\title{
Incidence of depression in Epilepsy patients
}

\section{DOI: http://dx.doi.org/10.4314/rj.v1i1.5F}

Vincent Sezibera, and Domitile Nyirasafari,

National University of Rwanda.

Correspondence Email : vsezibera@nur.ac.rw

\begin{abstract}
Epilepsy and depression are frequent pathologies especially in the developing countries where the level of health facilities and the standards of living are very low. Scholars have stated the relationship between epilepsy and depression but with less precision on symptoms and socio-demographic variables difference.

The study assessed the co-morbidity between the two different diagnostic entities, one neurologic (epilepsy) and the other psychiatric (depression). From a purposive sample of 392 people consulting for epilepsy, only 105 patients whose EEG diagnosis was positive were recruited in accordance with the inclusion criteria. Screening for depression, the Hamilton Rating Scale for Depression (HRSD) was used for the depression prevalence assessment. Results from the study showed that epilepsy patients are likely to present with depression symptoms with significant odds for all symptoms like depressive mood, insomnia, work and activities poor performance, anxiety, and somatic symptoms. Patients with generalized seizure are likely to suffer from severe comorbid depression as compared to patients with partial seizure.

Epilepsy treatment should incorporate depression diagnosis and management to ensure better management of the epilepsy burden.
\end{abstract}

Key words: Depression, Epilepsy, morbidity, diagnosis, Rwanda 


\section{Introduction}

Epilepsy is a neurological disorder marked by sudden recurrent episodes of sensory disturbance, loss of consciousness and convulsions, associated with abnormal electrical activity in the brain, i.e.; disruptions of the electrical activity in the brain (Musyimi, 2010). With regard the etiological factors; neurologists distinguish symptomatic epilepsy (i.e., from known neurological causes) and idiopathic epilepsy (i.e. from unknown causes) (Loiseau \& Jallon, 1979). In terms of its seizures, the classification includes partial and generalized seizures. Generally, the partial seizure epilepsy symptoms don't include consciousness impairment. However, generalized seizure symptoms include generalized convulsions, loss of consciousness and falling down, generalized body stiffening, violent jerking, followed by deep sleep (Musyimi, 2010). In addition to these acute symptoms, clinicians note also injuries and accidents, tongue biting and urinary incontinence. Repetitive and generalized seizures that characterize epilepsy are hypothesized to affect physically and psychologically the patients. Specific psychological negative outcomes include humiliation, shame, being depressed and increased feeling of impotence.

In Rwanda, epilepsy is reported to be the most frequently diagnosed neurological disorder as compared to other psychiatric and psychological disorders (Simms, Atijosan, Kuper, Nuhu, \& Rischewski, 2008). Policy makers in the health sector have pledged to develop a mental health national policy that includes epilepsy as a particular phenomenon in Rwanda (Ministry of Health, 2011).

To date, epilepsy diagnosis and treatment rely on a neurological model (EEG test) and thus the treatment includes anti-convulsive drugs. These drugs are intended to decrease the overwhelming and recurrent epileptic convulsions. However, scholars and practitioners have identified correlates between medical conditions and depression disorder (Narasimhan, Raynor, \& Jones, 2008). Thus, epileptics may suffer from both epilepsy and depression symptoms (Mendez, Cummings, \& Benson, 1986, Simms, Atijosan, Kuper, Nuhu, \& Rischewski, 2008). As a psychiatric disorder, depression is often extremely disabling and accompanied a sense of despair, hopelessness sometimes involves ruminations with ideas of 
culpability, shamefulness, and self- depreciation, which leads sometimes to suicide ideations and attempts (DSM IV, APA 1994). Previous studies have found that risk for suicide attempts in epileptics was 4 to 5 times greater as compared to the general population (Trimble \& Reynolds, 1987). In this regard, existing findings ascertained that $5 \%$ to $7 \%$ of epileptics attempted suicide using self-poisoning (Mackay, 1979; Hawton et al., 1980 ; Hollaway, 2005).

Although depression is often clinically present in patients consulting for their epilepsy, the treatment given is usually relegated to pharmacological treatments solely for epilepsy symptoms. However, untreated depression can affect the anti-epilepsy treatment compliance and thus defeat the recovery from epileptic convulsions. As a comorbid disorder to epilepsy, depression is hypothesized to affect the biological treatment of epilepsy (Kanner, 2009).

This study aimed at assessing the depression prevalence in epileptic patients in Rwanda. Moreover, the study screened for depression severity difference with regard socio-demographic variables (age, sex, education level) and seizures types (partial versus generalized seizures).

\section{Methods}

\section{Participants}

Participants were recruited from both inpatients and outpatients consulting with epileptic symptoms at Ruhengeri Hospital, Northern Province of Rwanda. Out of the 392 patients being seen for epilepsy, only $105(26.78 \%)$ met EEG criteria for an epilepsy diagnosis. The 105 participants met all the inclusion criteria and thus participated in the study. Inclusion criteria included (a) being treated for epileptic symptoms, (b) meeting EEG diagnostic indices for epilepsy established by medical professionals, (c) not presenting other medical condition established by medical professionals, (d) consent to participate in the research study, (e) being skilled in reading and writing Kinyarwanda (maternal language of the participants). All patients meeting inclusion criteria accepted to participate in this 
research. The Department of Clinical Psychology committee reviewed and approved the study design.

Aged from 9 to 68 years $(M=26.58 ; S D=12.76)$, the sample comprised participants slightly males (55.2\% males) and low level of education $(45.7 \%$ of sample have completed only primary school). Diagnostically, $56.2 \%$ of patients presented with generalized seizures while $43.8 \%$ presented with partial seizures.

\section{Measures}

\section{Epilepsy diagnosis}

The epilepsy diagnosis was based on clinical presentations and EEG findings from medical professionals. The EEG diagnosis is made from the electrical signals from the brain. The clinical findings specified whether seizures are generalized or partial.

\section{Depression screening}

The Hamilton Rating Scale for Depression (HRSD), 21 items and 0-4 Likert scale, was used for screening likely clinical cases of depression. The scale can be used as a continuous outcome for analyses or a cut point can be applied for the likely depression diagnosis), distinguishes levels of depression prevalence such as absent (0-9 total score), minor (10-19 total score), moderate (20-26) and severe (27 and above). The HRSD Kinyarwanda version (translated by the Neuro-Psychiatric Hospital CARAES-NDERA) presented reliable internal consistency $(\alpha=.96)$.

\section{Data management and analysis}

The SPSS package (18th version) was used for data analysis. Descriptive analysis was performed for HRSD categories and depression prevalence. For socio-demographic variables and depression differences, comparison analysis (independent t-test and ANOVA) were computed. Depression prevalence and severity were performed on each of the socio-demographic variables (age, sex and education level) and epileptic seizures (partial versus generalized seizures). 


\section{Results}

\section{Depression screening}

Data analysis revealed that $48.6 \%(\mathrm{~N}=105)$ of respondents met HRSD criteria for a depression diagnosis. From the epilepsy patients presenting depression symptoms, the majority was qualifying for a severe depression $(51 \%)$, whereas others were reporting symptoms ranging from moderate $(29.4 \%)$ to mild depression (19.6\%).

\section{Sex, Age and Level of Education moderating depression severity}

It was assumed that socio-demographic variables (sex, age and level of education) would predict depression prevalence in epilepsy patients. Comparing epilepsy patients with and without depression, statistical analysis (Cross tabulation under SPSS) revealed that sex $(\chi 2(3)=9.127 ; p<0.05)$, age $(\chi 2(3)=26.901 ; p<0.001)$ and level of education $(\chi 2(9)=69.163, \mathrm{p}<0.001)$ predicted the likelihood of depression severity. Comparison analysis (independent t-test and ANOVA) of HDRS total scores and socio-demographic variables confirmed significant age, sex and education level differences on depression scores, respectively $\mathrm{t}(103)=-4.359$, $\mathrm{p}<0.001, \mathrm{t}(103)=3.092, \mathrm{p}<0.01$ and $\mathrm{F}(3,101)=36.438 ; \mathrm{p}<0.001$. Epileptic adult females, with lower education level (illiterate), were the most vulnerable to depression.

\section{Depression prevalence as compared to epilepsy seizures}

EEG findings distinguished patients with partial seizures $(43.8 \%$, $\mathrm{N}=105)$ from those with generalized seizures (56.2\%, $\mathrm{N}=105)$. Comparison analysis revealed significant difference; patients with generalized seizure $(M=25.55 ; S D=19)$ scored high on HDRS total scores as compared to patients with partial seizure $(M=9.71$; $\mathrm{SD}=6.77), \mathrm{t}(103)=-5.261, \mathrm{p}<0.001$. All individuals meeting criteria for generalized seizure epilepsy were diagnosed with severe depression $(24.8 \%, \mathrm{~N}=105)$. Moreover, data analysis revealed that a majority of epilepsy patients diagnosed with generalized seizure and reporting severe depression, was female $(69.2 \%)$. Therefore, being female and having generalized seizure was found to be associated 
with increased risk of severe depression among epilepsy patients in this sample.

\section{Discussion and Conclusion}

This study aimed at assessing depression morbidity and severity in patients seeking care for epilepsy in a clinic setting in Rwanda. Indeed, studies have established the co-occurrence of psychiatric disorders in patients with medical conditions. In this regard, epileptics were hypothesized to present with depression as a comorbid psychiatric disorder to epilepsy.

Participants' recruitment for this study showed that out of the 392 patients consulting for their epilepsy, only 105 (26.78\%) met the neurological criteria (EEG findings) for epilepsy diagnosis. These figures indicate a real concern about epilepsy symptomatology and diagnosis in Rwanda. These results could relate to improper diagnosis including presentation of convulsive symptoms that are not pertaining to neurological epilepsy. Congruent with the second assumption, research and clinic findings stated the existence of psychogenic non-epileptic seizures (PNES) with refractory seizure disorder (Nezadal, Hovorka, Herman, Nemcova, Bajacek, \& Stichova, 2011). (Fadiman, 1997). Major PNES symptoms include initially closed eyelids, rapid tremor, asynchronous limb movement, preictal pseudosleep, and a side-to-side head movement (Nezadal et al., 2011). Also a number of psychiatric conditions including personality disorders, anxiety and depression have shown significant association with PNES. In this regard, the malingering convulsive symptoms may be related to psychological somatization rather than neurological epilepsy. Also, and given the magnitude of the 1994 genocide in Rwanda, there might be PTSD symptoms or TBI (Traumatic Brain Injury) outcomes that are misdiagnosed as neurological epilepsy.

Assessing depression prevalence, $48.6 \%(\mathrm{~N}=105)$ of the participants met the diagnostic criteria for likely depression. Screening for depression severity, $24.8 \%$ of patients reported severe depression symptoms. Considering the inter-correlation between age, sex and education level, results from this study highlighted that adult females with lower education level (illiterate) were more likely to present 
with severe depression. The finding is congruent with existing figures stating the positive correlation between the two disorders (Novy, et al., 2012) especially in developing countries (Simms, Atijosan, Kuper, Nuhu, \& Rischewski, 2008). In their cross-sectional study in the Republic of Benin (West Africa), Nubukpo, Houinato, Preux, Avode, \& Clement (2004) found that epileptics(196) also reported severe anxiety (79.8\%) and severe depression (89.6\%). Explaining the relationship between the epilepsy and the two psychiatric disorders severity, the researchers cited the social and cultural burden faced by epileptic patients in developing countries.

In the present study, adult females with a lower education level tended to report higher rates of severe depression compared to men. The explanation of such finding is twofold. With low level of education, and given the culture perception of the epilepsy in the Northern Province where participants were recruited, respondents in this study are hypothesized to have limited knowledge on epilepsy etiology and treatment that orient their subjection perception of the burden. This is consistent with research findings indicating positive correlation between education level and quality of life in female epileptics (Yue, et al., 2011). With regard culture influences and spiritual traditions, people in that specific region of Rwanda believe in supernatural forces, bewitchment or poisoning as causes of epilepsy (Fadiman, 1997). Therefore, patients believe that these uncontrollable forces are the source of their convulsion and that are inherited sometimes from parents to children. In this manner, having symptoms of epilepsy may be ascribed to spirit possession or other socially shameful conditions. In this regard, epilepsy is felt as unpredictable and untreatable which may further worsen ones subjective appraisal of the disease.

The gender differences observed in the present study were particularly striking. Several studies have found poorer quality of life in women with epilepsy as compared to men (Yue, et al., 2011; Alanis-Guevara, et al., 2005; Buck, Jacoby, Backer, Ley, \& Steen, 1999). This difference has been attributed to the social role played by women as compared to men, particularly in lower resource settings. Given the psychosocial problems resulting from epilepsy burden, women are exposed to tremendous negative consequences of the 
symptoms which are further associated with risk of mood disorders. Negative outputs include change in family functioning (e.g. being rejected and divorced), social stigma and rejection by family and community members. In most African communities, women occupy key position within the family and thus their important roles may also be poorly performed when suffering from epilepsy, leading to social consequences. Obviously, these social artifacts are assumed to influence the onset and development of depression symptoms associated with epilepsy.

Whereas previous studies demonstrated a correlation between depression and partial seizure (Mendez, Cummings, \& Benson, 1986), results from this study revealed that epileptics diagnosed with generalized seizure are the most vulnerable to severe depression. Scholars assessing the relationship between the types of seizure and depression prevalence have argued that in patients with temporal lobe epilepsy, anticonvulsive drugs and psychosocial factors increase the likelihood of depression occurrence (Yue, et al., 2011).

In addition to biological and physiological factors accounting for the correlation between seizure types and depression, it is clear that subjective appraisal of the symptoms associated with epilepsy and the side effect of the medication can contribute to the depression onset and development in epileptics. Moreover, the social context of epilepsy (i.e. the significance of symptoms, causal factors, treatment and prognosis) is assumed to contribute to the psychological distress, mainly depression and social isolation. Future research should explore the cultural factors and the role of spirituality influencing attitudes towards epilepsy and depression in culturally diverse settings such as the present research in Rwanda.

As a conclusion, this study has confirmed the presence of depression in people with epilepsy in Rwanda. The study also raises the issue of patients with non-epileptics convulsive symptoms under epilepsy treatment who may be misdiagnosed. It is however assumed that the non-epileptics convulsive symptoms are characterizing a somatization phenomenon since participants are recruited from a post-genocide country. With regard to epilepsy treatment, it should be noted that untreated depression runs the risk of negatively impacting epileptic treatment compliance. Thus, neurologists should 
screen for depression and should include depression diagnosis and treatment in their routine clinical treatment of epilepsy (Yue, et al., 2011).

\section{Limitations}

This study examined the relationship between depression and epilepsy. Nevertheless, the causal pathways by which the age, sex and education level affect the depression vulnerability in epileptics require further exploration. In the end, this sample is cross sectional and represents only referred patients seeking treatment for symptoms of epilepsy across northern districts in Rwanda. Future research might employ representative sampling and longitudinal data. In future research, it may prove further interesting to investigate mediation of socio-demographic variables between epilepsy and depression morbidity.

Also, in future research, epilepsy and patients history should be taken into consideration to better understand epilepsy and depression morbidity. Moreover, additional risk factors should be screened for and their effect on the actual depression severity such as the patients history of depression, seizure frequency and epilepy medication history. Chronic epilepsy and prescribed pharmacological treatment is assumed to generate depression symptoms as a side effect. Our results indicate that rather than as a side effect, epilepsy may often be co-morbid with depression and both conditions merit high quality care. Similarly, social perceptions of epilepsy and their influence on the course of depression over time require further examination in the context of Rwanda and other settings where both conditions are widely misunderstood. 


\section{References}

1. Alanis-Guevara, I., Pena, E., Corona, T., Lopez-Ayala, T., Lopez-Meza, E., \& Lopez-Gomez, M. (2005). Sleep disturbances, socioeconomic status, and seizure control as main predictors of quality of life in epilepsy. Epilepsy and Behaviour, 7, 481-485.

2. Buck, D., Jacoby, A., Backer, G., Ley, H., \& Steen, N. (1999). Cross-cultural differences in health-related quality of life of people with epilepsy:findings from a European study. Qual Life Res, 8(8), 675-685.

3. Fadiman, A. (1997). The spirit catches you and you fall down. New York: Farr, Straus and Giroux.

4. Halgin, R., \& Whitbourne, S. K. (2009). Abnormal psychology : Clinical Perspective on psychological disorders. McGraw Hill Humanities/Scial Sciences/Languages.

5. Hawton, K., Fagg, J., \& Marsack, P. (1980). Association between epilepsy and attempted suicide. J Neurol Neurosurg Psychiatry, 43, 168-170.

6. Hollaway, K.J. (2005). New research on epilepsy and behavior. Nova Biomedical Pub Inc.

7. Kanner, A. M. (2009). DEpression and epilepsy : Do glucocorticids and glutamate explain their relationship ? Current neurology and Neuroscience reports, 9(4), 304-312. doi:DOI: 10.1007/s11910-009-0046-1

8. Loiseau, P., \& Jallon, P. (1979). Les épilepsies. Paris: Masson.

9. Mackay, A. (1979). Self-poisoning-acomplicated of epilepsy. $\mathrm{Br}$ J. Psychiatry, 134, 277-282.

10. Mendez, M. F., Cummings, J. L., \& Benson, D. F. (1986). Depression in Epilepsy : Significance and phenomenology. Archives of Neurology, 43(8), 766-770.

11. Ministry of Health (2011). Mental health national policy in Rwanda. Kigali: unpublished .

12. Musyimi, D. N. (2010). Your A-Z on Mental Health. Nairobi.

13. Narasimhan, M., Raynor, J. D., \& Jones, A. B. (2008). Depression in the medically ill : Diagnostic and therapeutic implications. Current Psychiatry Reports, 272-279. 
14. Nezadal, T., Hovorka, J., Herman, E., Nemcova, I., Bajacek, M., \& Stichova, E. (2011). Psychogenic non-epileptic seizures: our video-EEG experience. Neurological Research, 33(7), 694-700. doi:10.1179/1743132811Y.0000000003

15. Novy, J., Castelao, E., Preisig, M., Marques, V. P., Waeber, G., Vollenweider, P., \& Rosetti, A. O. (2012). Psychiatric comorbidities and cardiovascular risk factors in people with lifetime history of epilepsy of an urban community. Clinical Neurology and Neurosurgery, 26-30. doi:10.1016/j.clineuro.2011.08.019

16. Nubukpo, P., Houinato, D., Preux, P., Avode, G., \& Clement, J. (2004). Anxiety and depression among the epileptics in general population in Benin (western Africa). Encephale-Revue de psychiatrie clinique biologique et thérapeutique, 201-219.

17. Sebera, F., \& Nyirazinyoye, H. (2006). Prevalence de l'épilepsie au Rwanda. Enquête sur la connaissance, perception, et attitudes de la population et des professionnels de la santé. Kigali: Unpublished.

18. Simms, V., Atijosan, O., Kuper, H., Nuhu, A., \& Rischewski, D. (2008). Prevalence of epilepsy in Rwanda : a national crosssectional survey. Tropical Medicine and International Health, 13(8), 1047-1053.

19. Trimble, H. R., \& Reynolds, E. (1987). Epilepsy, Behavior and Cognitive Fumction. New York: Brisbane.

20. Yue, L., Yu, P.-m., Zhao, D.-h., Wu, D.-y., Zhu, G.-X., Wu, X.y., \& Hong, Z. (2011). Determinants of quality of life in people with epilepsy and their gender differences. Epilepsy and Behavior, 22(4), 692-696. 